\title{
Electrical Polarization of Lead Bromide Crystals. II
}

\author{
J. SCHOONMAN AND F. G. DIJKMAN \\ Solid State Department, Physical Laboratory, University of Utrecht, Utrecht, The \\ Netherlands
}

Received October 26, 1971

\begin{abstract}
Electrical currents are measured as a function of time after application of a dc voltage on a cell arragement $+\mathrm{C} / \mathrm{PbBr}_{2} / \mathrm{C}-$. The presence of nonlinear polarization phenomena has been established from a test of Ohm's law, the superposition principle, and from initial and steady-state conductivities calculated from the polarization curves. In addition, nonlinear potential profiles were measured. The polarization effects are attributed to the build-up of space charge regions near the electrodes in view of the fact that the electrode process is slow compared to the velocity of the charge carriers in the bulk of the crystal. The steady-state current is limited by an electrode process with an activation enthalpy of $0.45 \pm 0.05 \mathrm{eV}$. The cathode is more effective in blocking than the anode.
\end{abstract}

\section{Introduction}

Electrolytical polarization of solids is due to a relative displacement of negative and positive charge, or to rotation of dipoles in that solid. When dipoles in the solid orient along the direction of an applied field the relative displacement is over a short distance. In that case the term dielectric relaxation is used, since the phenomenon is not connected with electrode processes. When charge carriers migrate through the solid under the influence of an electric field, but are blocked at an interface like a boundary in the crystal, or as is more frequently encountered at an electrode-crystal contact, space-charge polarization results. The mobile charge carrier under consideration may be either ionic or electronic. Examples of both kinds of polarization are known in quite a number of solids of very different nature (1).

During the last twenty years much attention has been devoted to space-charge effects in electronically conducting semiconductors, and the theory on that problem has progressed relatively far. In contrast little progress has been made in the theory of space-charge polarization in ionic conductors, neither in the build-up of the polarization, nor in the steady state. The treatment given by Jaffé and Le May (2), and its extension by Allnatt et al. (3) do not predict a current decay as is observed experimentally. (C) 1972 by Academic Press, Inc.
Its main striking feature is that a uniform field is assumed, which is in direct contradiction to the concept of space-charge. In the steady state the theories of McDonald (4-7) are pertinent, but mainly connected with problems encountered in semiconductors. Quantitatively McDonald's theories are hardly applicable to ionic conductors. They are even less applicable if in the steady state a current is observed, as a consequence of partially blocking electrodes.

We have found strong polarization phenomena in undoped and in thallium (I) bromide-doped lead bromide crystals. They are attributed to the build-up of a space-charge layer near the electrodes.

\section{Experimental}

The experiments were carried out on single crystals of lead bromide grown by a zonemelting technique $(8,9)$. The crystals were cleaved perpendicular to the $c$-axis. Usually surface areas of 0.5 to about $2.0 \mathrm{~cm}^{2}$, and a crystal thickness of 0.1 to about $0.3 \mathrm{~cm}$ were used in the experiments. Contact between the platinum electrodes and crystal surfaces was established with Aquadag. The measurements were carried out in vacuo in a conductivity cell as described previously $(10)$.

Direct current voltages applied to the crystals ranged from 0.07 to $0.85 \mathrm{~V}$, the decomposition 111 
voltage of lead bromide being $1.1 \mathrm{~V}$. Temperatures ranged from room temperature to $440 \mathrm{~K}$. Charge and discharge currents were measured with an autoranging Keithly picoammeter (model 445), which was commanded by a Solartron data transfer unit. Measurements could be made every one second, and at larger intervals up to two hours. The picoammeter never needed more than $0.5 \mathrm{~s}$ to make a reading with range changing, and usually, holding range, far less. The data transfer unit transferred time and current data to a tape punch (Facit 4070).

Alternating current conductivities here referred to were measured with a General Radio impedance bridge (type 1608A) with external oscillator (type 1210C), used at a frequency of $1 \mathrm{kc} / \mathrm{s}$, and an amplifier-null-detector (type 1232A).

The potential profile across the crystal was measured in a dc field at room temperature in air. A graphite-covered probe was pressed successively on a number of parallel Aquadag electrodes painted on one side of the crystal. The potential of the probe relative to one electrode was measured with a Keithley electrometer (610BR). Crystals with thickness up to $1 \mathrm{~cm}$ were used in these experiments.

\section{Results}

We studied current-time relations during the polarization of lead bromide crystals, pure as well as doped with thallium (I) bromide. In lead bromide Schottky defects dominate. Alternating current ionic conductivity studies have revealed that bromide ion vacancies are the mobile species (II).

The thallium (I) bromide-doped crystals show a wide temperature range in which the concentration of the bromide ion vacancies, $\left[V_{\mathrm{Br}}\right]$ is constant, since, because of the increased $\left[V_{\mathbf{B r}_{\mathbf{r}}}^{*}\right]$ the intrinsic bromide ion vacancy concentrations, $\left[V_{\mathrm{Br}}^{*}\right]_{0}$ can be neglected: moreover, ac conductivities in the temperature region involved show the absence of associated defects of the type $\left(\mathrm{Tl}_{\mathrm{Pb}} \cdot V_{\mathrm{Br}}\right)^{\times}$.

On application of a de voltage across the crystal, an initial current, $i_{0}$, appeared which was proportional to the applied field. It was difficult, however, to measure the initial currents with great accuracy, as the current decayed very rapidly after application of the field. The mean values of the initial current densities, divided by the initial field strength, were very close to the values of the ac conductivity, so that it is obvious that the current was transported through the crystal by the bromide ion vacancies.

In Fig. 1 we have plotted the initial parts of some polarization curves of undoped lead bromide. In Fig. 2 the charge and discharge curve for an undoped crystal at $353 \mathrm{~K}$ are shown. The steady-state currents, $i_{\sim}$, were reached after about one day. At low voltages $(E<0.45 V)$ the discharge currents were usually somewhat smaller than the charge currents, whereas above $0.45 \mathrm{~V}$ the reversed situation was observed.

The polarization and depolarization (discharge) currents did not obey Ohm's law very well. The calculated conductivities, i.e., the current density divided by the electric field strength, differed by as much as $10 \%$ at the same time for different voltages. Values for $i_{0} / i_{\sim}$ range from about $10^{2}$ in undoped lead bromide to $7 \times 10^{2}$ in TIBr-doped lead bromide, which is more due to the large $i_{0}$ differences between undoped and TIBr-doped lead bromide, than between the observed values for $i_{\sim}$. In fact, the values for $i_{\sim}$ all lie in the same range for different crystals under identical circumstances. The steady-state current shows a linear dependence on the cathodic surface area of the crystal, and is proportional to the reciprocal of the thickness of the crystal. It is exponentially dependent both on applicd voltage and reciprocal temperature. Figure 3 shows the dependence on voltage at several temperatures. Extrapolated values for zero voltage were plotted as $\log i_{\sim}$ versus $1 / T$. An activation enthalpy of $0.45 \pm 0.05 \mathrm{eV}$ was found, which is a significantly higher value than the activation enthalpy for the migration of the bromide ion vacancies, for which the value $0.25 \pm 0.01 \mathrm{eV}$ was found (10).

From measurements on the electromotive force built up in the crystal in the steady state, it turned out that only very small parts of the applied voltage appeared as potential drops between the graphite electrodes and the crystal surface. A typical measurement showed that, when $0.143 \mathrm{~V}$ was applied, the emf of the crystal was $0.135 \mathrm{~V}$.

We measured potential profiles on various crystals. A few of such profiles are presented in Fig. 4. The abscissa is normalized with respect to the thickness of the crystal, whereas the ordinate is normalized with respect to the applied voltage. In most of the profiles the largest potential drop appeared at the cathode. Reversing the polarity of the applied voltage (Fig. $4 \mathrm{a}$ and b) 


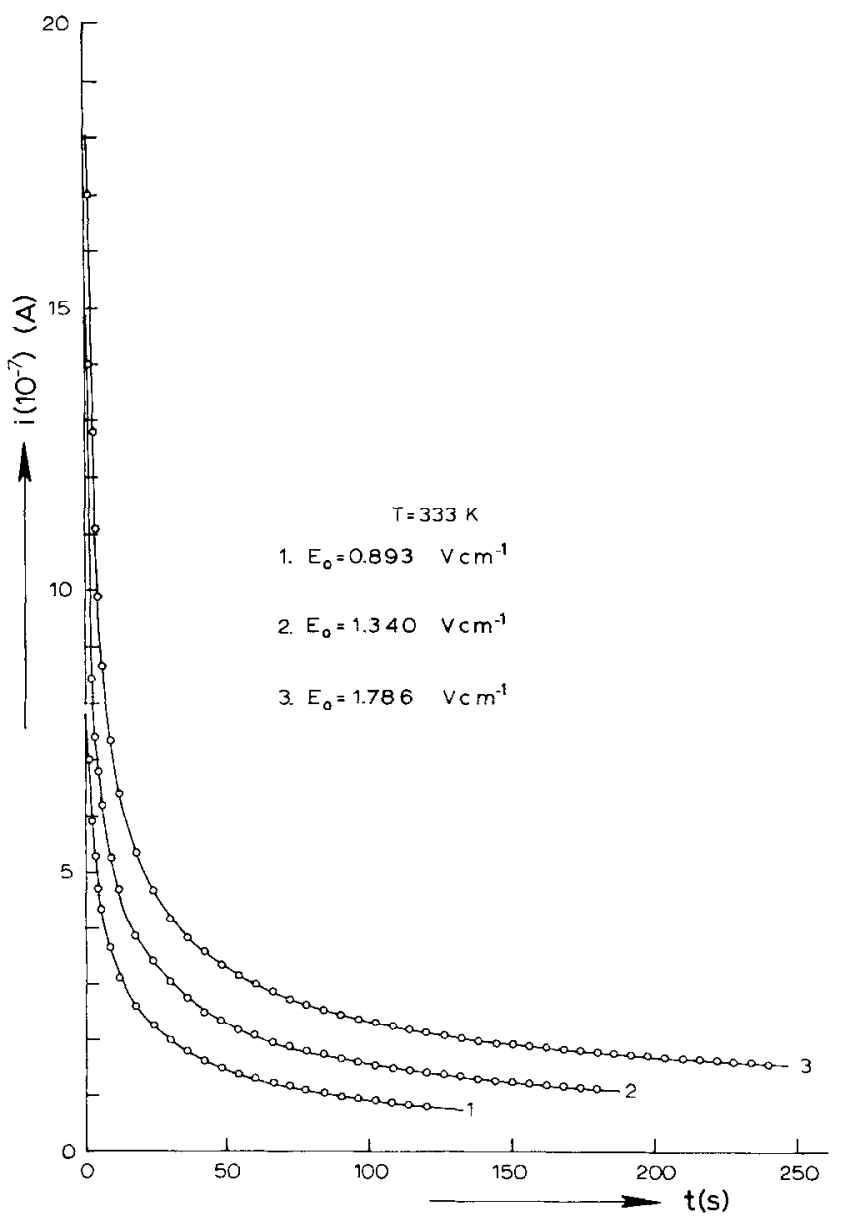

FIG. 1. Polarization currents in undoped lead bromide at $T=333 \mathrm{~K}, d=0.224 \mathrm{~cm}$.

causes the asymmetric potential profile to reverse, which indicates that the asymmetry is not due to local variations in dielectric properties (15).

\section{Discussion}

The nonlinear polarization phenomena encountered in the dc experiments on lead bromide in a cell arrangement $+\mathrm{C} / \mathrm{PbBr}_{2} / \mathrm{C}$ - must be attributed to a build-up of space-charge containing regions near the graphite electrodes $(13,16)$. This conclusion is based upon the following features: (a) the conductivity calculated at $t_{0}(t=0)$ equals the true ionic conductivity whereas $\sigma_{\sim}$, calculated from the steady state is much smaller than the true ionic conductivity, (b) the superposition principle which states that the charge current $i_{p}(t)$, obtained by subtracting the steady-state current, $i_{\sim}$, from the polarization current, $i(t)$, equals the discharge current, $\left|i_{d}(t)\right|$, does not hold, (c) Ohm's law is not valid for intermediate values of $i(t)$ and for $i_{\sim}$, and (d) the potential profiles indicate a strongly nonlinear electric field. It must be pointed out that no quantitative value can be attributed to the profiles, since they could only be measured in air at room temperature.

Theoretical progress in the build-up of spacecharge in ionic crystals is rather poor. In 1952 Jaffé and Le May (2) solved the nonlinear differential equations by assuming a constant field throughout the crystal $(d E / d \mathrm{x}=0]$. They further neglected the formation and recombination of charge carriers, but did not regard this as a serious deficiency. The solutions of the linearized differential equations given by Jaffé and Le May are represented by a sum of exponentials decaying with time. Agreement with the experiments on $\mathrm{KCl}$ of Allnatt et al. (3), who applied the theory of 


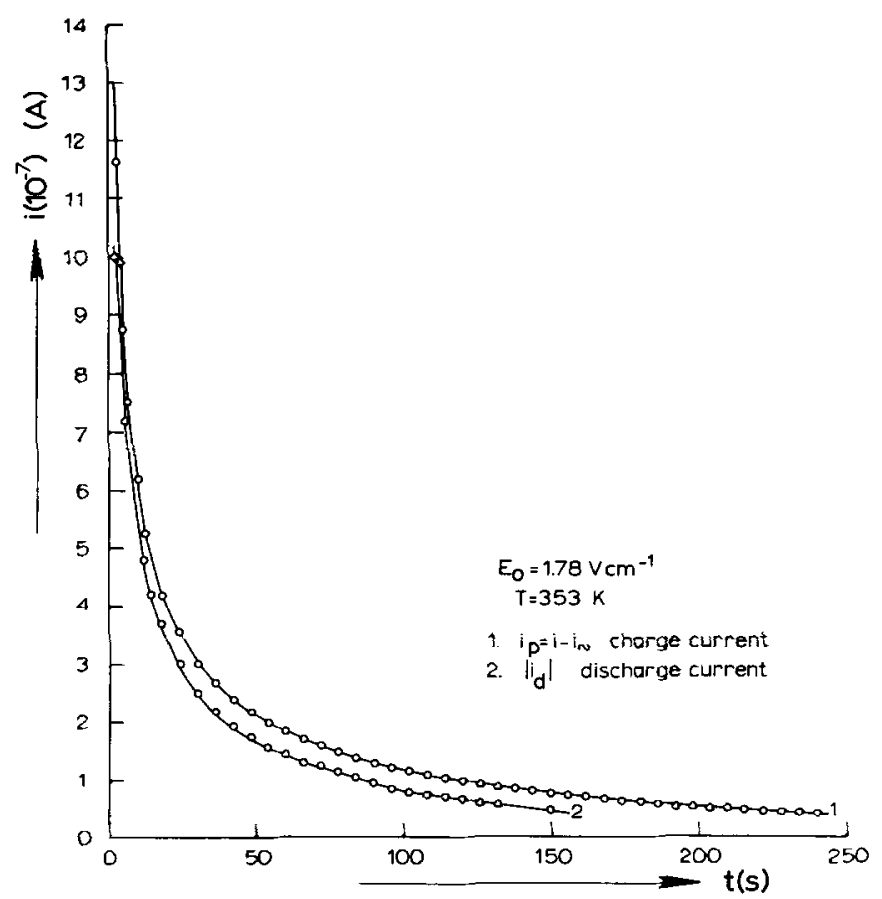

FIG. 2. Charge and discharge curves for undoped lead bromide at $T=353 \mathrm{~K}, d=0.224 \mathrm{~cm}$.

Jaffé and Le May is poor, however, The first $150 \mathrm{~s}$ of their current-time plots can be resolved in two exponentials, but the theoretically calculated

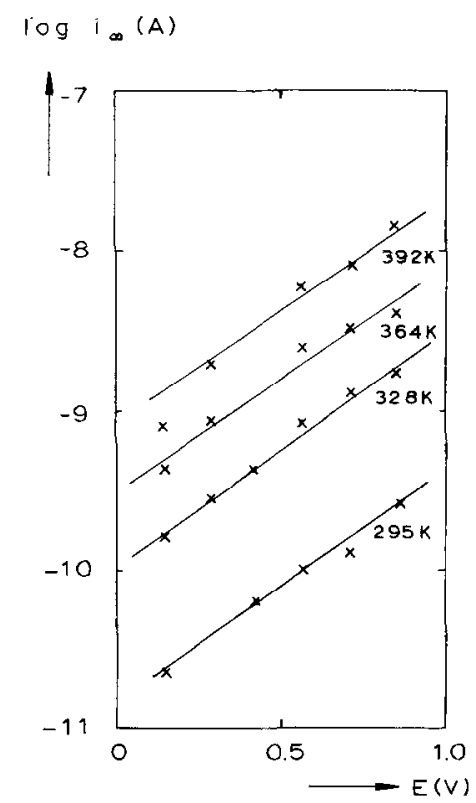

Fig. 3. Steady-state currents in a thallium (I) bromidedoped lead bromide crystal $(d=0.286 \mathrm{~cm})$, plotted as $\log i \sim$ versus $E$. exponents differ by a factor of 10 from those found in the actual experiments.

We analyzed the current-time plots in the same way as Allnatt et al. (3) did in their polarization studies on $\mathrm{KCl}$. The results are exemplified in Figs. 5 and 6 for the first $200 \mathrm{~s}$. The charge current can be expressed by

$$
i_{p}(t)=A e^{-\alpha t}+B e^{-\beta t}
$$

The values calculated theoretically for the reciprocal time constants $\alpha$ and $\beta$ are smaller than the experimental values by about a factor of $10^{3}$. The values for $\alpha$ and $\beta$ decrease with increasingly applied voltage, $E$, and with increasing temperature. As was pointed out by Allnatt et al. (3) $\beta$ should vary as $E^{2}$, while the temperature coefficient of $\beta$ should be that of the diffusion coefficient of the bromide ion vacancies. Neither of these predictions, originating from the Jaffé theory agrees with our results, which must probably be due to the incorrectness of the constant field approximation.

For polarization times from 10 to $10^{4} \log -\log$ graphs of the current-time data show straight lines with a slope that is close to the value -0.5 . For times shorter than 10 , and larger than $10^{4} \mathrm{~s}$ the deviation became pronounced. If we express the charge current as $i_{p}(t)=A t^{-\alpha}$ with 


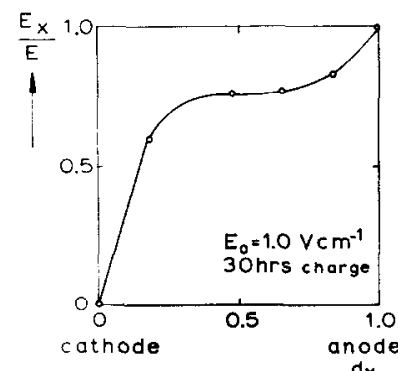

(a)

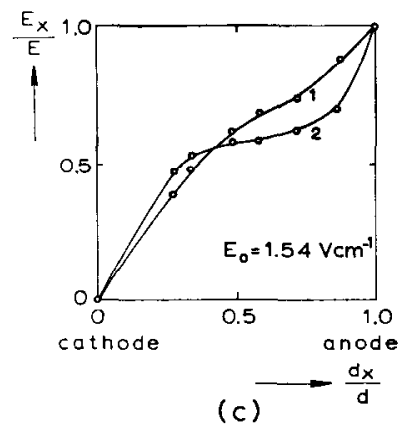

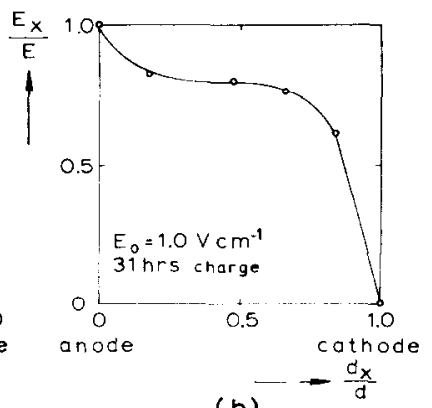

(b)

FIG. 4. Dependence on normalized thickness $\left(d_{\times} / d\right)$ of normalized voltage $\left(E_{\times} / E\right)$ : (a) steady-state situation $(d=$ $0.440 \mathrm{~cm}$ ), (b) the same situation as in (a), but with reversed polarity, (c) build-up of space-charge: (1) 30 min charge, (2) 17 h charge $(d=0.650 \mathrm{~cm})$.

$\alpha \sim 0.5$, which is a dependence frequently encountered in polarization experiments $(13,14)$, it is obvious that this expression cannot hold for $t_{0}$, since it would indicate an infinite current: moreover it cannot hold at extremely long times, since the integral $\tilde{\int}_{0} i_{p} d t$ has no finite value for

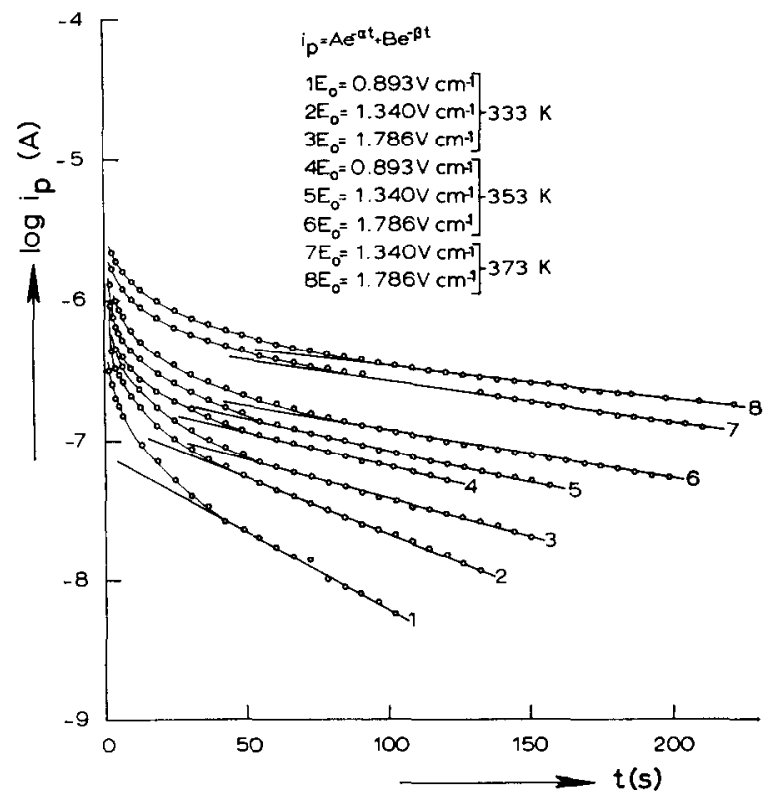

FIG. 5. Plots of the charge current $i_{p}=i-i_{\sim}$ of undoped lead bromide as $\log i_{p}$ versus $t$ for the first $200 \mathrm{~s} . d=0.224$ cm (cf. Fig. 1). 


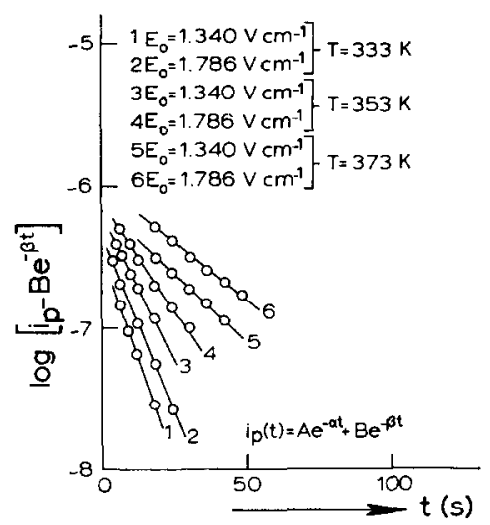

Fig. 6. Analysis of the charge current as the sum of two exponential terms (cf. Figs. 1 and 5).

infinite times, whereas the passed charge obviously has a finite value. The former objection can be overcome by expressing the current as

$$
i_{p}(t)=\left(A(t+1)^{-\frac{1}{2}}\right.
$$

the latter, however, remains. In the experiments we always measured lower currents after $10^{4} \mathrm{~s}$ than indicated by this formula (cf. Fig. 7). The constant $A$ can be determined, since conductivities calculated from the initial currents closely resembles the true ionic conductivity, turning Eq. (2) into

$$
i_{p}(t)=\left[V_{\mathrm{Br}}^{\cdot}\right] e \mu_{V_{\mathrm{Br}}^{\cdot}}(O / d) E(t+1)^{-\frac{1}{2}}
$$

where $\left[V_{\mathrm{Br}}^{*}\right]$ is the bromide ion vacancy concentration, $\mu_{V_{\mathrm{Br}}}$ their mobility, $O$ the crystal surface, $d$ the crystal thickness, $e$ the electronic charge and $E$ the applied voltage.
The discharge currents are also straight lines in a $\log -\log$ graph between $t=10$ and $t=10^{4} \mathrm{~s}$. The slope varied around the value -0.5 by no more than $10 \%$.

The steady-state current can be attributed to either slow electrolysis of the crystal, or to an electronic current through the crystal, or to a mixture of both. The voltage dependence of the steady-state current suggests the former process (17). This process can be represented by the reactions

$$
\mathrm{Br}_{\mathrm{Br}}^{x} \rightarrow \frac{1}{2} \mathrm{Br}_{2}(\mathrm{~g})+V_{\mathrm{Br}}^{*}+e^{\prime} \quad \text { (anode) }
$$

and

$$
\mathrm{Pb}_{\mathrm{Pb}}^{\times}+2 V_{\mathrm{Br}}^{\cdot}+2 e^{\prime} \rightarrow \mathrm{Pb}(\mathrm{s}) \quad \text { (cathode). }
$$

The charge transport through the crystal is due to migration of bromide ion vacancies. The steady-state current is limited by an electrode process with an activation enthalpy of $0.45 \pm$ $0.05 \mathrm{eV}$. This enthalpy probably determines the reaction velocity of the slowest step of some electrode process for the nature of which we can think of many possibilities. From the dependence of $i_{\sim}$ on the cathodic surface area it would seem that the cathode is more effective in blocking than the anode is. The experimental asymmetric potential profiles support this idea. It is obvious from the results presented in this paper, and the associated work in this field that further theoretical and experimental work is required before the mechanism underlying the space-charge polarization in lead bromide can be confidently established. Such work is now in progress in our laboratory.

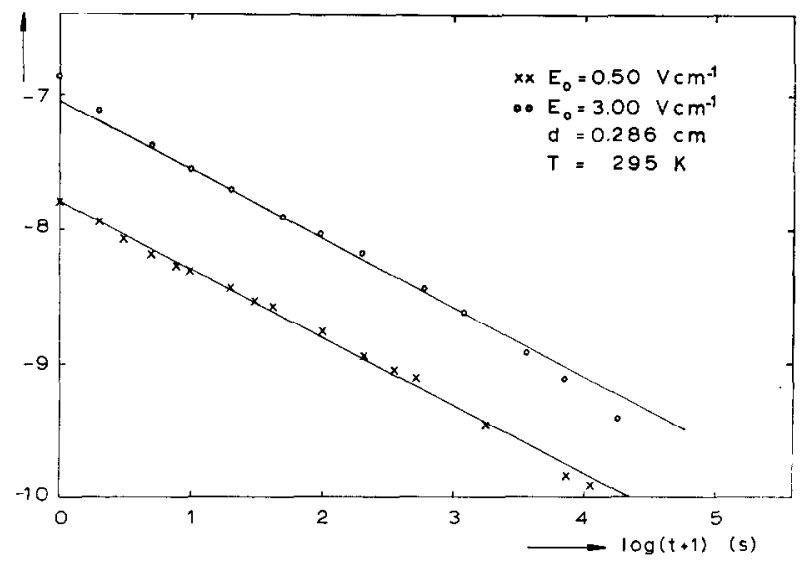

FIG. 7. Examples of an analysis of the charge current according to Eq. (2) for the first $10^{4} \mathrm{~s}$. $\mathrm{PbBr}_{2}-\mathrm{TlBr}$ with thickness $d=0.286 \mathrm{~cm}$. 


\section{Acknowledgments}

The authors are grateful to Prof. Dr. I. H. van Santen and Prof. Dr. G. Blasse for encouraging this work and for stimulating discussions. They would like to thank Dr. G. H. J. Broers for profitable discussions.

\section{References}

1. F. A. KRöGER, "The Chemistry of Imperfect Crystals," pp. 914-921. North-Holland, Amsterdam, 1964.

2. G. Jaffé ANd Ch. Le May, J. Chem. Phys. 21, 920 (1953).

3. A. R. Allnatt, P. W. M. Jacobs, and J. N. Maycock, J. Chem. Phys. 43, 2526 (1965).

4. J. R. McDonald and M. K. Brachman, J. Chem. Phys. 22, 1314 (1954).

5. J. R. McDonald, J. Chem. Phys. 22, 1317 (1954).

6. J. R. McDonald, J. Chem. Phys. 29, 1346 (1958).
7. J. R. McDonald, J. Chem. Phys. 30, 806 (1959).

8. J. F. Verwey and J. Schoonman, Physica 35, 286 (1967).

9. J. F. VERWEY, thesis, University of Utrecht, 1967.

10. J. Schoonman, J. Solid State Chem., in press. 5, 62 (July, 1972)

11. J. Schoonman And J. F. Verwey, Physica 39, 244 (1968).

12. A. F. JofFé, "The Physics of Crystals." McGraw-Hill, New York, 1928.

13. P. H. Sutter And A. S. Nowick, J. Appl. Phys. 34, 734 (1963).

14. L. B. Harris, J. Phys. Chem. Solids 32, 59 (1971).

15. R. Solomon, A. Sher, ANd M. W. Muller, J. Appl. Phys. 37, 3427 (1966).

16. C. A. Bucci ANd S. A. Riva, J. Phys. Chem. Solids 26, 363 (1965).

17. P. W. M. JACOBS AND J. N. MaYcock, J. Chem. Phys. 39, 757 (1963). 